\title{
Impact of diabetes mellitus on long-term outcome after unstable angina and non-ST-segment elevation myocardial infarction treated with a very early invasive strategy
}

\author{
C. Müller ${ }^{1,2}$ • F. J. Neumann ${ }^{1}$ M. Ferenc ${ }^{1}$ A. P. Perruchoud ${ }^{2}$ H. J. Büttner ${ }^{1}$ \\ 1 The Heart Center Bad Krozingen, Germany \\ ${ }^{2}$ Departement Innere Medizin, Medizinische Universitätsklinik Basel, Basel, Switzerland
}

\begin{abstract}
Aims/hypothesis. We sought to evaluate the impact of diabetes mellitus on long-term outcome in patients with unstable angina and non-ST-segment elevation myocardial infarction treated with a very early invasive strategy.

Methods. We carried out a prospective cohort study in 270 diabetic and 1163 non-diabetic patients with unstable angina and non-ST-segment elevation myocardial infarction. All patients underwent coronary angiography and, if appropriate, subsequent revascularisation within 24 hours of admission. The primary endpoint was allcause mortality during follow-up for up to 60 months. Results. Diabetic patients had less favourable baseline characteristics including more advanced coronary artery disease and more severe unstable angina and nonST-segment elevation myocardial infarction. Percutaneous coronary intervention was performed in $53 \%$ of diabetic patients and $56 \%$ of non-diabetic patients. Coronary artery bypass grafting was done in $21 \%$ of diabetic
\end{abstract}

patients and $12 \%$ of non-diabetic patients. In-hospital mortality (4.1\% vs $1.3 \%$; hazard ratio 3.47 ; $95 \%$ CI: 1.57 to $7.64 ; p=0.002)$ and long-term mortality $(9.7 \%$ vs $4.9 \%$; hazard ratio $2.11 ; 95 \%$ CI: 1.33 to 3.36 ; $p=0.002$ ) were significantly higher in diabetic patients. After adjustment for differences in baseline characteristics, diabetes mellitus was no longer an independent predictor of long-term mortality (hazard ratio 1.43; 95\% CI: 0.74 to $2.78 ; p=0.292$ ).

Conclusions/interpretation. Diabetic patients treated with a very early invasive strategy for unstable angina and non-ST-segment elevation myocardial infarction have a higher in-hospital and long-term mortality that is largely explained by their less favourable baseline characteristics including more advanced coronary artery disease and more severe unstable angina and nonST-segment elevation myocardial infarction.

Keywords Diabetes mellitus · Long-term mortality · Myocardial infarction - Revascularisation - Unstable angina
Received: 8 March 2004 / Accepted: 22 April 2004

Published online: 3 July 2004

(C) Springer-Verlag 2004

C. Müller $(\bowtie)$

Departement Innere Medizin,

Medizinische Universitätsklinik Basel, Petersgraben 4,

4031 Basel, Switzerland

E-mail: chmueller@uhbs.ch

Tel.: +41-61-2652525, Fax: +41-61-2655353

Abbreviations: CK, creatine phosphokinase · FRISC, Fragmin and fast Revascularisation during InStability in Coronary artery disease · OASIS, Organisation to Assess Strategies for Ischemic Syndromes - TACTICS-TIMI 18, Treat angina with Aggrastat and determine Cost of Therapy with an Invasive or Conservative Strategy-Thrombolysis In Myocardial Infarction 18 - UA/NSTEMI, unstable angina and non-ST-segment elevation myocardial infarction

\section{Introduction}

Diabetes mellitus is an important risk factor for coronary artery disease, which is associated with increased mortality and morbidity in diabetic patients as compared with non-diabetic patients [1, 2, 3]. Patients with diabetes mellitus with no history of coronary artery disease have the same risk of cardiovascular death as non-diabetic patients with a history of myocardial infarction [4]. In addition, patients with diabetes mellitus have not experienced the reduction in cardiovascular mortality rates that has recently been observed in non-diabetic subjects [5]. The number of adults with diabetes mellitus worldwide is likely to grow from 135 million in 1995 to approximately 300 million in 2025 [6]. However, only limited informa- 
tion is available regarding the outcome of diabetic patients after hospital admission with unstable angina and non-ST-segment elevation myocardial infarction (UA/NSTEMI), the most common reason for admission to a coronary care unit $[7,8,9,10,11]$. The OASIS registry prospectively collected data from UA/NSTEMI patients treated with predominately medical therapy in six different countries including the United States, and reported that diabetes mellitus independently predicted mortality [11]. Overall, cumulative mortality in patients with diabetes mellitus was $19 \%$ at 24 months in the OASIS registry.

Revascularisation, irrespective of the primary success of medical therapy, has become the preferred treatment strategy in patients with UA/NSTEMI [12, $13,14]$. Current practice guidelines for the management of patients with UA/NSTEMI from the American College of Cardiology, the American Heart Association, and the European Society of Cardiology recommend an early invasive strategy for most patients $[15$, 16]. The long-term outcome of patients with diabetes mellitus and UA/NSTEMI treated with a very early invasive strategy remains to be assessed. We sought to determine the long-term outcome of systematic revascularisation within 24 hours in diabetic patients. In addition, we evaluated whether the prognostic impact of diabetes mellitus is different between women and men.

\section{Subjects and methods}

Study population. From January 1996 to December 1999, consecutive patients admitted to our centre with UA/NSTEMI were treated with a very early invasive strategy. Patients were eligible for inclusion in this study if they underwent coronary angiography for symptoms of myocardial ischaemia occurring at rest (Braunwald class IIIB unstable angina) [17]. We excluded patients who had the following conditions: (i) a first case of angina pectoris during exertion, or worsening angina during exertion (Braunwald class 1A-C); (ii) persistent ST-elevation; or (iii) postinfarction angina (Braunwald class 1C, 2C, 3C). Also excluded were patients in whom angiography was not performed due to patient refusal $(n=6)$ or extremely severe concomitant disease $(n=9$ with severe dementia or advanced malignancy). The study was carried out in accordance with the Declaration of Helsinki and approved by the institutional review board. Informed consent was obtained from all participating patients. Diabetes was defined as the patient having a known history of diabetes mellitus treated currently with either diet intervention, oral glucose-lowering agents or insulin. Irrespective of the presence or absence of diabetes, strict glycaemic control was observed in all patients. Upon hospital discharge, all patients were instructed to follow a low-cholesterol diet, and statins were recommended to achieve an LDL cholesterol lower than $2.6 \mathrm{mmol} / \mathrm{l}$ during follow-up. All patients received aspirin and beta blockers unless contraindicated.

Very early invasive strategy. Patients with persistent chest pain underwent immediate coronary angiography. In patients asymptomatic while on medical therapy, coronary angiography was performed within 24 hours of admission. Whenever possible, coronary stenting of the culprit lesion was done directly after angiography. Stenting was not restricted to patients with one- and two-vessel disease, but also favoured in patients with three-vessel disease, if the target lesion seemed accessible. If percutaneous coronary intervention was not possible (unprotected left main disease, diffuse three-vessel disease) but revascularisation seemed necessary, patients were scheduled for urgent coronary artery bypass grafting.

Follow-up. All patients were scheduled for outpatient visits at 6 months. In addition, patients were contacted by questionnaire in September 2000, 4 years and 9 months after enrolment of the first patient. For patients reporting cardiac symptoms, at least one clinical and ECG examination was performed in the outpatient clinic or by the referring physician. All information derived from contingent hospital re-admission records and that provided by the referring physician or by the outpatient clinic was reviewed and entered into the computer database.

Endpoints and statistical analysis. The pre-specified primary endpoint was defined as death from all causes. As secondary endpoints we assessed non-fatal myocardial infarction and the composite of death and non-fatal myocardial infarction. Myocardial infarction was defined as typical chest pain at rest followed by an increase in creatine phosphokinase (CK and CK-MB more than two times the upper limit of normal, and more than five times the upper limit of normal after coronary artery bypass grafting) or new Q waves in the ECG. To meet this endpoint criterion, patients who initially had myocardial infarction had to develop ST-segment changes and an increase in CK of at least $50 \%$ over the previous trough level in at least two samples reaching at least three times the upper limit of normal.

Follow-up events were adjudicated by a clinical events committee. All patients received ECG recordings directly after percutaneous coronary intervention or coronary artery bypass grafting and on the following morning. In addition, cardiac markers (CK and CK-MB) were determined between 8 and 24 hours after the intervention, and additionally whenever ischaemic symptoms developed. The statistical analyses were performed using the SPSS/PC (version 11.0; SPSS, Chicago, Ill., USA) software package. Discrete variables were expressed as percentages $(95 \% \mathrm{CI})$ and continuous variables were expressed as means $\pm \mathrm{SD}$. A $p$ value of 0.05 or less was considered statistically significant. For the rarer outcomes, confidence intervals were calculated using the Wilson method. Comparisons were made using ANOVA for independent samples and chi square tests as appropriate. All hypothesis testing was two-tailed. Cox proportional hazards regression analysis was used as the appropriate method throughout. Multivariate Cox regression analysis was performed to identify independent predictors of death. Baseline characteristics, ECG findings, markers of inflammation and myocardial necrosis, and the angiographic extent of coronary artery disease were entered into the model. The cumulative survival curves were constructed by the Kaplan-Meier method.

\section{Results}

Baseline characteristics. There were 270 patients with diabetes and 1163 patients without diabetes in this study. The vast majority of diabetic patients were classified as having Type 2 diabetes (261 of the 270, 97\%). Besides diet and exercise, therapy included insulin in $74(28 \%)$ of the 261 patients, and glibenclamide in 50 (19\%) of the 261 patients. Baseline characteristics 
Table 1. Baseline patient characteristics

\begin{tabular}{|c|c|c|c|}
\hline & Diabetic patients $(n=270)$ & Non-diabetic patients $(n=1163)$ & $p$ value \\
\hline Age (years) & $68 \pm 9$ & $64 \pm 11$ & $<0.001$ \\
\hline Female sex & $31(25-36)$ & $28(26-31)$ & 0.407 \\
\hline Previous coronary bypass grafting & $16(11-20)$ & $13(11-15)$ & 0.339 \\
\hline Previous coronary angioplasty & $22(17-27)$ & $22(20-25)$ & 0.858 \\
\hline Hypercholesterolaemia & $64(58-69)$ & $66(63-69)$ & 0.451 \\
\hline Angina pectoris at rest $>48 \mathrm{~h}$ & $16(12-20)$ & $17(15-19)$ & 0.616 \\
\hline Angina pectoris at rest $<48 \mathrm{~h}$ & $67(61-73)$ & $69(66-72)$ & 0.522 \\
\hline Non-Q-wave myocardial infarction & $17(13-22)$ & $14(12-16)$ & 0.167 \\
\hline Cardiopulmonary resuscitation & $3.0(0.9-5.0)$ & $0.9(0.4-1.5)$ & 0.009 \\
\hline Defibrillation (only) & $2.6(0.7-4.5)$ & $1.5(0.8-2.3)$ & 0.238 \\
\hline Cardiogenic shock & $1.1(0-2.4)$ & $0.9(0.3-1.4)$ & 0.695 \\
\hline Creatinine $(\mu \mathrm{mol} / \mathrm{l})$ & $91 \pm 74$ & $80 \pm 59$ & 0.016 \\
\hline White blood cell count $\left(\times 10^{3} / \mu \mathrm{l}\right)$ & $9.0 \pm 3.0$ & $8.8 \pm 8.0$ & 0.820 \\
\hline Platelet count $\left(\times 10^{3} / \mu \mathrm{l}\right)$ & $236 \pm 94$ & $235 \pm 70$ & 0.874 \\
\hline
\end{tabular}

Data are expressed as means $\pm \mathrm{SD}$, or percentages $(95 \% \mathrm{CI})$

Table 2. Baseline angiographic and procedural characteristics

\begin{tabular}{llll}
\hline & Diabetic patients $(n=270)$ & Non-diabetic patients $(n=1163)$ & $p$ value \\
\hline Coronary vessels with $\geq 50 \%$ stenosis & & & 0.001 \\
0 & $7(4-10)$ & $26(24-29)$ & 0.001 \\
1 & $19(14-24)$ & $24(21-26)$ & 0.023 \\
2 & $21(16-26)$ & $35(32-38)$ & 0.367 \\
3 & $53(46-59)$ & $56(53-59)$ & 0.001 \\
Percutaneous coronary intervention & $53(47-59)$ & $81(78-84)$ & 0.278 \\
Proportion with stent & $74(66-82)$ & $11(9-14)$ & 0.094 \\
Proportion with abciximab & $12(6-18)$ & $31(29-34)$ & 0.931 \\
Coronary artery bypass grafting & $21(17-26)$ & & 0.001 \\
Medical therapy & $26(21-31)$ & & 0.075 \\
\hline
\end{tabular}

Data are expressed as percentages $(95 \% \mathrm{CI})$

were considerably different between diabetic and nondiabetic patients (Tables 1 and 2). Diabetic patients were older, more often hypertensive and less often smokers, and they more frequently had a previous myocardial infarction as well as cardiac arrest during the UA/NSTEMI leading to admission than non-diabetic patients. They also had a higher incidence of new ST-segment depression and raised troponin T.

Angiographic characteristics and revascularisation. Coronary angiography revealed that coronary artery disease was more extensive in diabetic patients. More than half of the patients with diabetes mellitus had three-vessel disease compared with $35 \%$ of patients without diabetes mellitus. Conversely, non-diabetic patients were two times as likely to have no coronary vessel with a high-grade lesion as diabetic patients. Ac- cordingly, coronary artery bypass grafting was chosen significantly more often $(21 \%$ vs $12 \%, p=0.001)$ in patients with diabetes. However, percutaneous coronary intervention was the predominant revascularisation procedure in both groups. Percutaneous coronary intervention was performed in $53 \%$ of patients with diabetes and in $56 \%$ of patients without diabetes. Altogether, two-thirds of patients underwent revascularisation.

In-hospital and long-term outcome. Patients with diabetes mellitus had a significantly higher in-hospital mortality rate than patients without diabetes mellitus $(4.1 \%$ vs $1.3 \%$; hazard ratio 3.47 ; $95 \%$ CI: 1.57 to 7.64$)$. In addition, non-fatal Q-wave myocardial infarction and the combined endpoint of death or myocardial infarction was recorded more frequently during the initial hospitalisation in patients with diabetes mellitus (Table 3 ). 
Table 3. Association between diabetes mellitus and outcome

\begin{tabular}{|c|c|c|c|c|}
\hline & $\begin{array}{l}\text { Diabetic patients } \\
(n=270)\end{array}$ & $\begin{array}{l}\text { Non-diabetic patients } \\
(n=1163)\end{array}$ & $\begin{array}{l}\text { Hazard ratio } \\
(95 \% \mathrm{CI})\end{array}$ & $p$ value \\
\hline \multicolumn{5}{|l|}{ In-hospital } \\
\hline Death & $4.1(2.1-7.2)$ & $1.3(0.7-2.1)$ & $3.47(1.57-7.64)$ & 0.002 \\
\hline Death or myocardial infarction ${ }^{\mathrm{a}}$ & $7.8(4.9-11.6)$ & $3.7(2.7-4.9)$ & $2.41(1.40-4.15)$ & 0.002 \\
\hline Q-wave myocardial infarction & $1.1(0.2-3.2)$ & $0.3(0.1-0.8)$ & $4.44(0.90-21.99)$ & 0.068 \\
\hline Death & $9.6(6.4-13.8)$ & $4.9(3.7-6.3)$ & $2.11(1.33-3.36)$ & 0.002 \\
\hline Death or myocardial infarction ${ }^{\mathrm{a}}$ & $13.7(9.8-18.4)$ & $8.6(7.1-10.4)$ & $1.76(1.20-2.59)$ & 0.004 \\
\hline Myocardial infarction & $4.4(2.3-7.6)$ & $4.0(3.0-5.3)$ & $1.21(0.62-2.36)$ & 0.570 \\
\hline
\end{tabular}

Data are expressed as percentages $(95 \% \mathrm{CI})$ unless otherwise stated. ${ }^{\text {a }}$ Counting only one event per patient

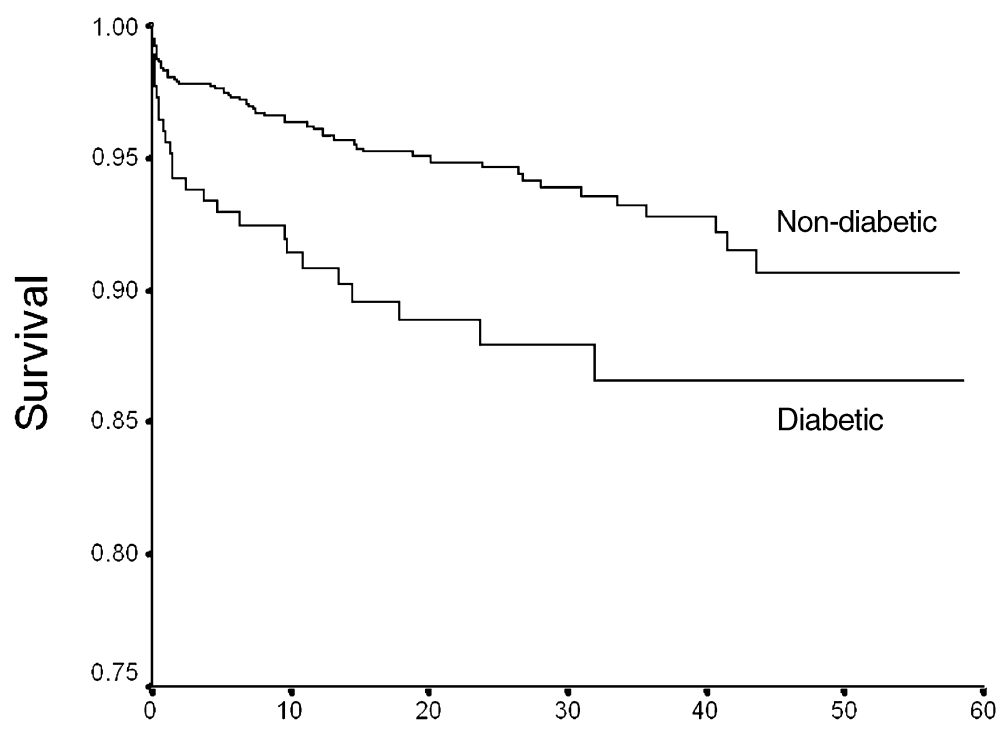

Months

$\begin{array}{rrrrrrl}1163 & 763 & 518 & 306 & 169 & 50 & \text { Non-diabetic (no. at risk) } \\ 270 & 170 & 107 & 68 & 34 & 13 & \text { Diabetic (no. at risk) }\end{array}$

Fig. 1. Cumulative survival in diabetic and non-diabetic patients during long-term follow-up. $p=0.0016$ by log-rank

The mean interval between admission and last patient contact or death was 19 months in diabetic patients and 20 months in non-diabetic patients. During follow-up, mortality was significantly higher in patients with diabetes mellitus than in patients without diabetes mellitus (Fig. 1). Kaplan-Meier analysis revealed a cumulative survival rate of $86.6 \%$ for diabetic patients and $92.8 \%$ for non-diabetic patients at 36 months. The incidence of non-fatal myocardial infarction was similar in the two groups.

Association between diabetes mellitus and sex. As shown in Figures 2 and 3, the impact of diabetes mellitus on long-term outcome was considerably dif- ferent between men and women ( $p=0.001$ for association). While diabetic men showed a marked reduction in survival rate compared with men without diabetes ( $83.7 \%$ vs $92.4 \%$ at 36 months), diabetic women showed a very similar survival rate to women without diabetes mellitus (93.9\% vs $93.7 \%$ at 36 months).

Diabetes mellitus and very early percutaneous coronary intervention. The long-term survival rate in patients undergoing very early revascularisation with percutaneous coronary intervention was excellent, irrespective of the presence or the absence of diabetes mellitus (Fig. 4). At 36 months, the cumulative survival rate was $92.4 \%$ in diabetic patients and $92.0 \%$ in non-diabetic patients.

Multivariate Cox regression analysis. After adjustment for baseline characteristics, ECG findings, mark- 


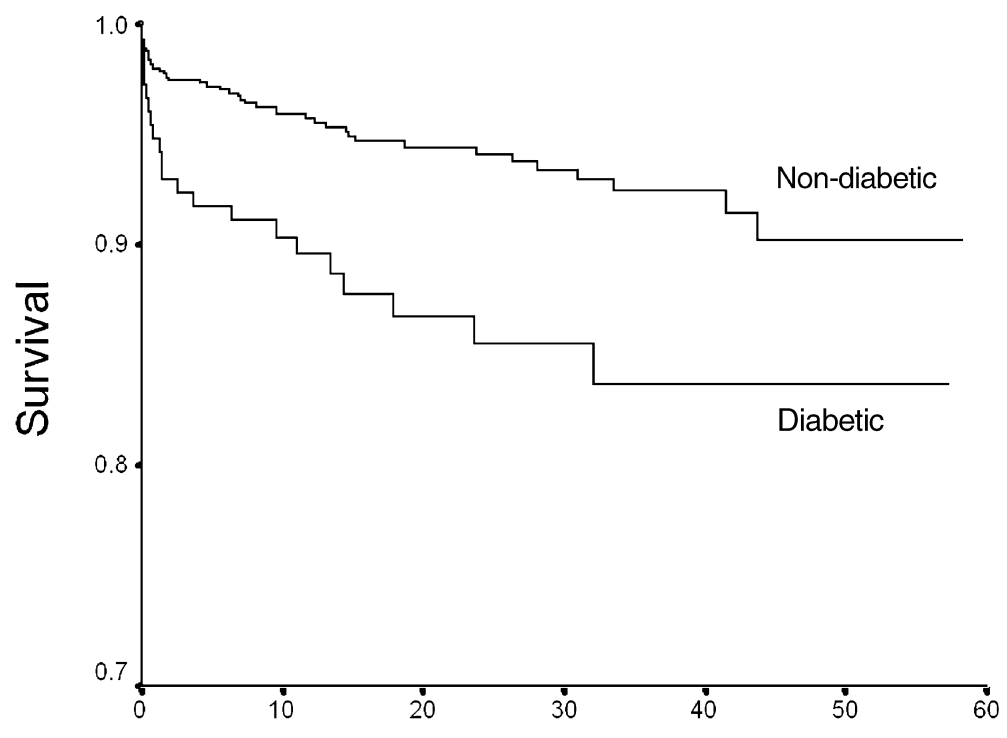

Months

\begin{tabular}{|c|c|c|c|c|c|}
\hline 539 & 359 & 214 & 114 & 34 & Non-diabetic (no. at risk) \\
\hline 122 & 74 & 51 & 24 & 9 & Diabetic (no. at risk) \\
\hline
\end{tabular}

Fig. 2. Cumulative survival in diabetic and non-diabetic men. $p=0.0010$ by log-rank

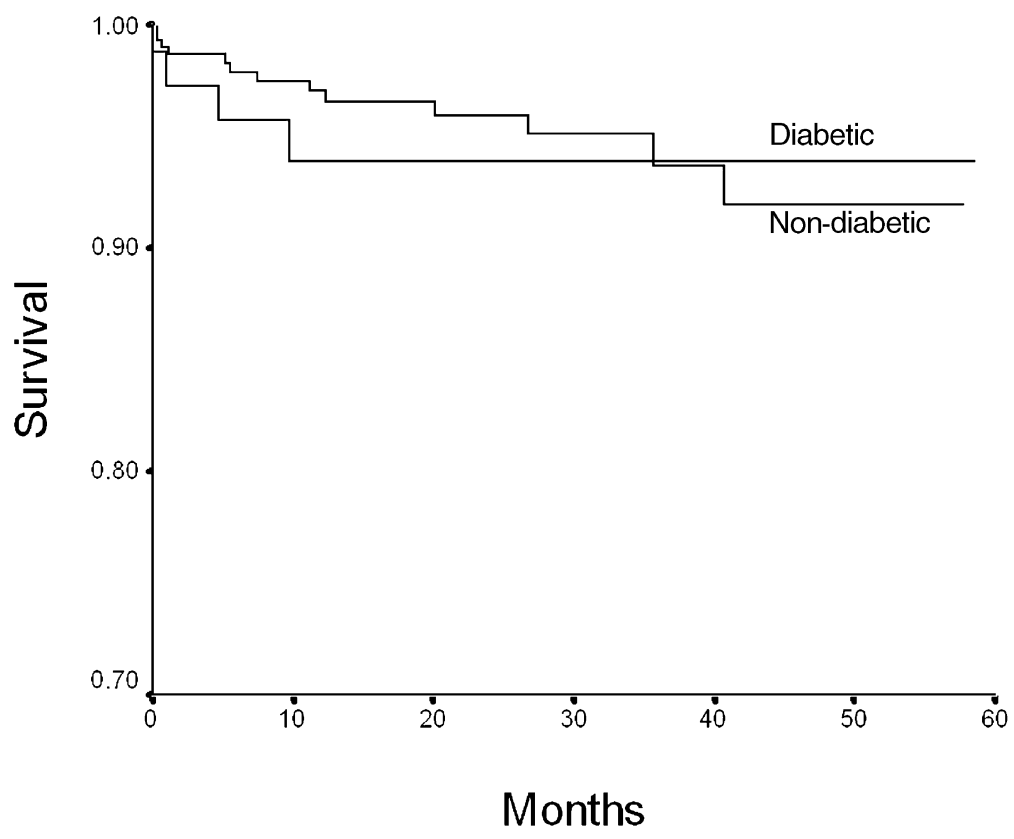

$\begin{array}{rrrrrrl}328 & 224 & 159 & 92 & 54 & 16 & \text { Non-diabetic (no. at risk) } \\ 82 & 48 & 33 & 17 & 10 & 4 & \text { Diabetic (no. at risk) }\end{array}$

Fig. 3. Cumulative survival in diabetic and non-diabetic women. $p=0.5792$ by $\log$-rank

ers of inflammation and myocardial necrosis, and the angiographic extent of coronary artery disease, diabetes mellitus was not found to be a significant independent predictor of long-term mortality (hazard ratio 1.43 ; $95 \%$ CI: 0.74 to $2.78 ; p=0.292$ ). Adding very early percutaneous coronary intervention as a variable to the multivariate Cox regression analysis showed that very early percutaneous coronary intervention was independently associated with a favourable outcome (hazard ratio for long-term mortality $0.50 ; 95 \%$ CI: 0.30 to $0.85 ; p=0.011)$. This association would persist if the analysis was restricted to diabetic patients only (hazard ratio 0.47 ; $95 \%$ CI: 0.20 to 1.09 ; $p=0.078$ ). 


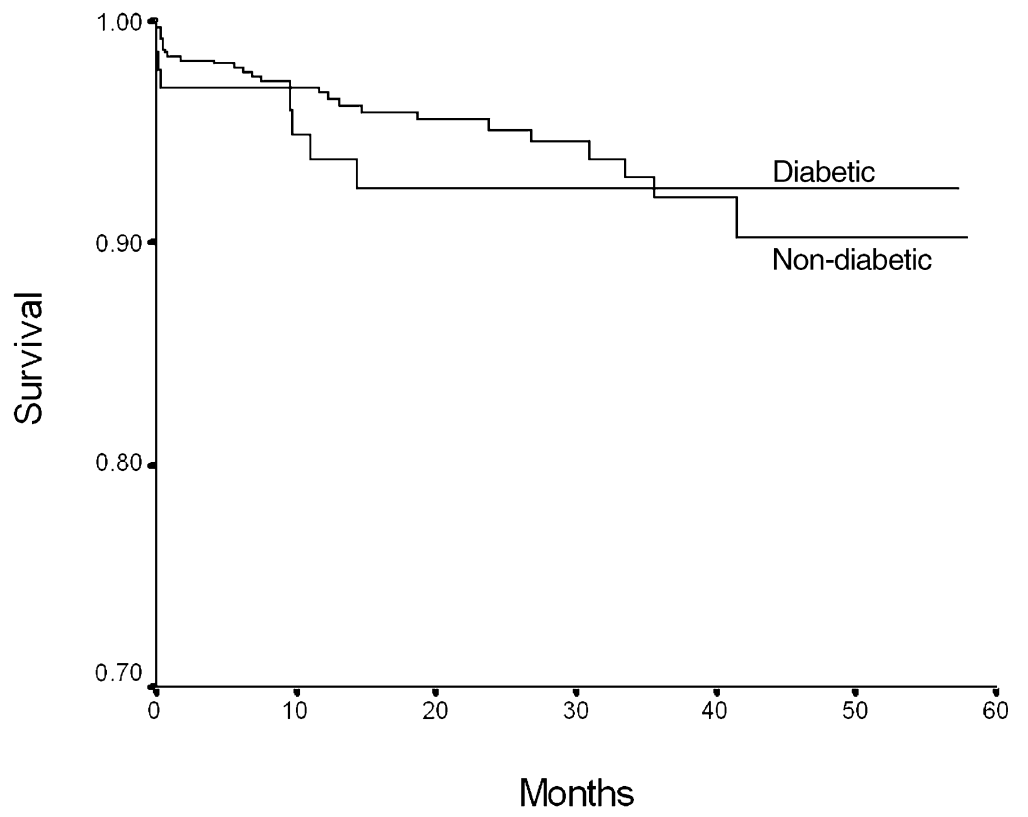

$\begin{array}{rrrrrrl}654 & 399 & 245 & 137 & 64 & 20 & \text { Non-diabetic (no. at risk) } \\ 140 & 88 & 48 & 29 & 10 & 4 & \text { Diabetic (no. at risk) }\end{array}$

Fig. 4. Cumulative survival in diabetic and non-diabetic patients revascularised very early with percutaneous coronary intervention. $p=0.4242$ by log-rank

\section{Discussion}

Our study of 1433 consecutive patients with UA/ NSTEMI treated with a very early invasive strategy showed that in-hospital and long-term mortality were significantly higher in patients with diabetes. This finding is consistent with previous studies that primarily used a conservative management strategy for UA/NSTEMI [7, 8, 9, 10, 11]. Nevertheless, as our major novel finding we were able to attribute most of this excess risk to two factors: more advanced coronary artery disease and more severe UA/NSTEMI at presentation. In fact, we could demonstrate that after adjusting for cofounders, diabetes mellitus was no longer an independent predictor of adverse outcome with this aggressive revascularisation strategy. We found important differences in baseline characteristics between diabetic and non-diabetic patients. Most importantly, diabetic patients were older and more often had previous myocardial infarction and three-vessel disease. In addition, the UA/NSTEMI seemed to be more severe in diabetic patients. Cardiopulmonary resuscitation, ST-segment depression and elevated troponin $\mathrm{T}$ were seen more often in diabetic patients than in non-diabetic patients. When we adjusted for these differences between diabetic and non-diabetic patients, the presence or absence of diabetes mellitus was no longer predictive of survival. As coronary angiography was performed in all patients, this study was able to include the angiographic extent of coronary artery disease in the multivariate model to assess independent predictors of long-term mortality. We found that the association of three-vessel disease and diabetes constitutes an important component of the predictive power of diabetes by univariate analysis.

This was not a randomised trial evaluating the very early invasive strategy. Accordingly, any comparison of the outcome observed in this prospective trial with historical controls has inherent limitations and should be interpreted with considerable caution. Although very similar with respect to patient baseline characteristics and study period, our study population experienced merely two-thirds the mortality at 24 months of that observed in the OASIS registry [11]. In our study, particularly in the patients revascularised very early with percutaneous coronary intervention, there was an excellent outcome irrespective of the presence or absence of diabetes mellitus. In fact, in the subgroup receiving very early percutaneous coronary intervention, long-term survival was identical in diabetic and nondiabetic patients. This observation is supported by subgroup analyses of the TACTICS-TIMI 18 and FRISC II studies [12, 13], in which diabetic patients showed a more pronounced benefit from the early invasive strategy than non-diabetic patients. In the TACTICS-TIMI 18 study, the early invasive strategy reduced the primary endpoint of death, non-fatal myocardial infarction, or re-hospitalisation, for an acute coronary syndrome at 6 months, from $27.7 \%$ to $20.1 \%$ in diabetic patients, and from $16.4 \%$ to $14.2 \%$ in nondiabetic patients. Our finding, however, can only be used to generate hypotheses and needs to be confirmed by further study. 
Excessive restriction during coronary angiography and revascularisation procedures may have contributed significantly to the dismal prognosis of patients with diabetes mellitus in earlier reports $[5,7,11]$. In a case-control study of 162 diabetic patients with unstable angina, diabetic patients less frequently underwent coronary angiography and angioplasty [7]. This observation was confirmed in the large OASIS registry. Although the diabetic patients in the registry were at clearly higher risk, having had more previous cardiovascular events including myocardial infarction, congestive heart failure and coronary artery bypass grafting, the rate of coronary angiography (37\% vs $37 \%$ ) and revascularisation by percutaneous coronary intervention or coronary artery bypass grafting (23\% vs $20 \%$ ) was not appropriately increased in patients with diabetes compared with patients without diabetes. In marked contrast, $70 \%$ of patients underwent revascularisation in this study. It is possible that at least partly as a result of this difference, 2-year mortality in patients with diabetes in the OASIS registry was increased by $50 \%$ compared with diabetic patients in our study. This hypothesis is supported by a recent study of 376 consecutive patients [5] showing that although diabetic patients with non-Q-wave myocardial infarction represented a cohort with more risk factors for poor outcome, aggressive in-hospital revascularisation with percutaneous coronary intervention resulted in excellent short-term outcome and 1-year survival similar to that in non-diabetic patients $(92 \%$ vs $94 \%$, NS). In addition, the beneficial effect of coronary stenting compared with standard balloon angioplasty on vessel patency and long-term clinical outcome in diabetic patients has now been demonstrated conclusively [18].

We acknowledge that the revascularisation strategy applied in UA/NSTEMI patients with three-vessel disease is controversial. In this study, coronary stenting was not restricted to patients with one- and two-vessel disease, but also favoured in patients with three-vessel disease, if the target lesion seemed accessible. It is important to note that the benefit of percutaneous coronary intervention over coronary artery bypass grafting in UA/NSTEMI patients with diabetes and three-vessel disease has yet to be proven. Although this intervention is common in well-established centres, which report an association with significantly lower morbidity, long-term outcome data from randomised trials specifically including UA/NSTEMI patients are lacking. In diabetic patients presenting with stable coronary artery disease, coronary artery bypass grafting seems to be more effective [19].

The treatment strategy applied in our patients is novel in two ways: the timing of the intervention and the preferred method of revascularisation. Coronary angiography and subsequent coronary stenting of the culprit lesion, as the primary revascularisation method, were performed within 24 hours of admission in all patients. The percutaneous coronary intervention : coronary artery bypass grafting ratio was $4: 1$. In the FRISC II study, coronary angiography was performed within the first 7 days and the percutaneous coronary intervention : coronary artery bypass grafting ratio was 1:1. In addition, the FRISC II study excluded patients with previous coronary artery bypass grafting, and patients with advanced age. These differences may explain the discrepant findings in multivariate analysis with respect to the independent impact of diabetes mellitus on long-term mortality [13]. In the FRISC II study, diabetes mellitus remained an independent predictor of mortality at 12 months.

The prognostic impact of diabetes mellitus was different between women and men. Women with diabetes mellitus had similar long-term outcome to women without diabetes mellitus, whereas mortality was significantly increased in diabetic men compared with non-diabetic men. This finding is in contrast to the observation of the OASIS registry, where diabetes mellitus was of greater prognostic importance for women than for men. This may suggest that the association between diabetes mellitus and sex depends on the management strategy applied in UA/NSTEMI (very early invasive vs conservative strategy). The very early invasive strategy seems to counteract and compensate for many of the extra risk factors present in diabetic women with UA/NSTEMI. The timing of the revascularisation procedure may, at least in part, explain why diabetic women derived such a major benefit from the management strategy applied in our study compared with the management strategy applied in OASIS. Although several pathophysiological distinctions [20, 21, 22, 23, 24] have been reported between women and men, the fact that women seem to form fewer coronary collaterals than men [24] may be of great importance. Due to this lack of collaterals, women may benefit particularly from a rapid correction of the epicardial obstruction during UA/NSTEMI [25]. However, further study is necessary to confirm this hypothesis.

Our analysis has four distinguishing features: (i) it is derived from a prospective study of consecutive unselected patients rather than from a randomised trial. This eliminates selection bias and allows generalisation and extrapolation of the findings into clinical practice; (ii) it involves long-term follow-up; (iii) a uniform revascularisation strategy was applied in all patients; and (iv) the extent of coronary artery disease was quantified in all patients and included in the multivariate analysis as a potential cofounder. This enables us to investigate the impact of diabetes mellitus, independent of the extent of coronary artery disease at presentation.

In conclusion, diabetic patients with UA/NSTEMI have more advanced coronary artery disease and more severe UA/NSTEMI than non-diabetic patients. Consequently, their in-hospital and long-term mortality is higher. However, when a very early invasive strategy 
is applied, and after adjustment for baseline characteristics, ECG findings, markers of inflammation and myocardial necrosis, and the angiographic extent of coronary artery disease, diabetes mellitus is no longer an independent predictor of death.

Acknowledgements. The results of this study were presented in part at the 52 ${ }^{\text {nd }}$ Annual Scientific Session of the American College of Cardiology, Chicago, 30 March-2 April 2003. The study was supported by research grants from the Swiss National Science Foundation, the Novartis Foundation, the Krokus Foundation, and the University of Basel (all to C. Müller). The authors wish to thank U. Keller for providing expert advice.

\section{References}

1. Abbott RD, Donahue RP, Kannel WB, Wilson PW (1988) The impact of diabetes on survival following myocardial infarction in men vs. women: the Framingham Study. JAMA 260:3456-3460

2. Butler WJ, Osrander LD Jr, Carman WJ, Lamphiear DE (1985) Mortality from coronary heart disease in the Tecumseh Study: long-term effect of diabetes mellitus, glucose intolerance, and other risk factors. Am $\mathrm{J}$ Epidemiol 121:541-547

3. Mueller C, Mc Hodgson JB, Brutsche M et al. (2002) Impact of intracoronary ultrasound guidance on long-term outcome of percutaneous coronary interventions in diabetics-insights from the randomised SIPS trial. Swiss Med Wkly 132:279-284

4. Haffner SM, Lehto S, Rönnemaa T, Pyörälä K, Laakso M (1998) Mortality from coronary heart disease in subjects with type 2 diabetes and in nondiabetic subjects with and without prior myocardial infarction. $N$ Engl J Med 339:229-34

5. Gu K, Cowie CC, Harris MI (1999) Diabetes and decline in heart disease mortality in US adults. JAMA 281:12911297

6. King H, Aubert RE, Herman WH (1998) Global burden of diabetes, 1995-2025: prevalence, numerical estimates, and projections. Diabetes Care 21:1414-1431

7. Fava S, Azzorpadi J, Agius-Muscat H (1997) Outcome of unstable angina in patients with diabetes mellitus. Diabet Med 14:209-213

8. Wilcox I, Freedman SB, Allman KC et al. (1991) Prognostic significance of a predischarge exercise test in risk stratification after unstable angina pectoris. J Am Coll Cardiol 18:667-683

9. Calvin JE, Klein LW, Van den Berg BJ et al. (1995) Risk stratification in unstable angina: prospective validation of the Braunwald classification. JAMA 273:136-141

10. Gowda MS, Vacek JL, Hallas D (1998) One-year outcomes of diabetic versus nondiabetic patients with non-Q-wave acute myocardial infarction treated with percutaneous transluminal coronary angioplasty. Am J Cardiol 89:10671071

11. Malmberg K, Yusuf S, Gerstein HC et al. (2000) Impact of diabetes on long-term prognosis in patients with unstable angina and non-Q-wave myocardial infarction. Results of the OASIS (Organization to assess strategies for ischemic syndromes) registry. Circulation 102:1014-1019

12. Cannon CP, Weintraub WS, Demopoulos LA et al. (2001) Comparison of early invasive and conservative strategies in patients with unstable coronary syndromes treated with the glycoprotein Iib/IIIa inhibitor tirofiban. N Engl J Med 344:1879-1887

13. Norhammar A, Malmberg K, Diderholm E et al. (2004) Diabetes mellitus: the major risk factor in unstable coronary artery disease even after consideration of the extent of coronary artery disease and benefits of revascularization. J Am Coll Cardiol 43:585-591

14. Neumann FJ, Kastrati A, Pogatsa-Murray G et al. (2003) Evaluation of prolonged antithrombotic pretreatment ("cooling-off strategy") before intervention in patients with unstable coronary syndromes. A randomized controlled trial. JAMA 290:1593-1599

15. Braunwald E, Antman EM, Beasley JW et al. (2002) ACC/AHA 2002 guideline update for the management of patients with unstable angina and non-ST-segment elevation acute myocardial infarction: a report of the American College of Cardiology/American Heart Association Task Force on Practice Guidelines (Committee on the Management of Patients with Unstable Angina). Available from http://www.acc.org/clinical/guidelines/unstable/unstable.pdf, accessed 1 February 2004

16. The Task Force on the Management of Acute Coronary Syndromes of the European Society of Cardiology (2002) Management of acute coronary syndromes in patients presenting without ST-segment elevation. Eur Heart J 23:1809-1840

17. Braunwald E (1989) Unstable angina: a classification. Circulation 80:410-414

18. Van Belle E, Périé M, Braune D et al. (2002) Effects of coronary stenting on vessel patency and long-term clinical outcome after percutaneous coronary revascularization in diabetic patients. J Am Coll Cardiol 40:410-417

19. Detre KM, Guo P, Holubkov R et al. (1999) Coronary revascularization in diabetic patients: a comparison of the randomized and observational components of the Bypass Angioplasty Revascularization Investigation (BARI). Circulation 99:633-40

20. Conlan MG, Folsom AR, Finch A et al. (1993) Associations of factor VIII and von Willebrand factor with age, race, sex, and risk factors for atherosclerosis: the Atherosclerosis Risk in Communities (ARIC) Study. Thromb Haemost 70:380-385

21. Tracy RP, Bovill EG, Fried LP et al. (1992) The distribution of coagulation factors VII and VIII and fibrinogen in adults over 65 years: results from the Cardiovascular Health Study. Ann Epidemiol 2:509-519

22. Stegnar M, Pentek M (1993) Fibrinolytic response to venous occlusion in healthy subjects: relationship to age, gender, body weight, blood lipids and insulin. Thromb Res 69:81-92

23. Johansson S, Bergstrand R, Schlossman D, Selin K, Vedin A, Wilhelmsson C (1984) Sex differences in cardioangiographic findings after myocardial infarction. Eur Heart J $5: 374-381$

24. Levenson J, Pessana F, Gariepy J, Armentano R, Simon A (2001) Gender differences in wall shear-mediated brachial artery vasoconstriction and vasodilation. J Am Coll Cardiol 38:1668-1674

25. Mueller C, Neumann FJ, Roskamm H et al. (2002) Women do have an improved long-term outcome after non-ST-elevation acute coronary syndromes treated very early and predominately with percutaneous coronary intervention: a prospective study in 1450 consecutive patients. J Am Coll Cardiol 40:245-250 